\title{
Fatores de risco associados à prematuridade e baixo peso ao nascer nos extremos da vida reprodutiva em uma maternidade privada
}

\author{
Risk factors associated with prematurity and low birth weight at the extremes of \\ reproductive life in a private maternity
}

Factores de riesgo asociados con la prematuridad y el bajo peso al nacer en los extremos de la vida reproductiva en una maternidad privada

Aurimery Gomes Chermont ${ }^{1}$, Elisa Figueiredo Alves da Silva ${ }^{1}$, Carinne Costa Vieira ${ }^{1}$, Luiz Euclides Coelho de Souza Filho², Erica Silva de Souza Matsumura ${ }^{2}$, Katiane da Costa Cunha ${ }^{2 *}$.

\section{RESUMO}

Objetivo: A presente pesquisa objetivou avaliar os fatores de risco associados à prematuridade e baixo peso ao nascer dos recém-nascidos das parturientes nos extremos da vida reprodutiva. Métodos: Participaram do estudo 1.117 prontuários de gestantes. Utilizou-se um protocolo de pesquisa previamente formulado pelos pesquisadores para registro das características sociodemográficas maternas, bem como dados sobre: paridade; idade gestacional; realização de pré-natal; intercorrências; via de parto; e outros. Resultados: Os achados evidenciaram que no grupo de mães acima de 34 anos a ocorrência de menos de seis consultas no pré-natal e o índice de Apgar no quinto minuto menor que sete predizem maiores chances de prematuridade e baixo peso ao nascer. Conclusão: Conclui-se que a idade materna influencia significativamente na ocorrência de parto prematuro e baixo peso ao nascimento, sendo justificado essencial políticas de saúde estaduais mais operante em relação ao pré-natal de qualidade.

Palavras-chave: Idade materna, Recém-nascido, Peso ao nascer, Gestantes, Parto.

\begin{abstract}
Objective: This research aimed to evaluate the risk factors associated with prematurity and low birth weight of parturient newborns at the extremes of reproductive life. Methods: The study included 1,117 medical records of pregnant women. A research protocol previously formulated by the researchers was used to record maternal sociodemographic characteristics, as well as data on parity; gestational age; prenatal care; complications; route of delivery; and others. Results: The findings showed that in the group of mothers older than 34 years, the occurrence of less than six prenatal consultations and the fifth minute Apgar score less than seven predict higher chances of prematurity and low birth weight. Conclusion: It is concluded that maternal age significantly influences the occurrence of preterm birth and low birth weight, being essential justifying more active state health policies in relation to quality prenatal care.
\end{abstract}

Keywords: Maternal age, Newborn, Birth weight, Pregnant women, Childbirth.

\section{RESUMEN}

Objetivo: Esta investigación tuvo como objetivo evaluar los factores de riesgo asociados con la prematuridad y el bajo peso al nacer de los recién nacidos parturientes en los extremos de la vida reproductiva. Métodos: El estudio incluyó 1.117 registros médicos de mujeres embarazadas. Se utilizó un protocolo de investigación previamente formulado por los investigadores para registrar características sociodemográficas maternas, así como datos sobre: paridad; edad gestacional; cuidado prenatal; complicaciones ruta de entrega; y otra. Resultados: Los resultados mostraron que en el grupo de madres mayores de 34 años, la ocurrencia de

${ }^{1}$ Universidade Federal do Pará (UFPA), Belém- Pará.

2Universidade do Estado do Pará (UEPA), Belém- Pará. *E-mail: katianefisio@yahoo.com.br 
menos de seis consultas prenatales y el puntaje de Apgar en el quinto minuto menos de siete predicen mayores posibilidades de prematuridad y bajo peso al nacer. Conclusión: Se concluye que la edad materna influye significativamente en la aparición de parto prematuro y bajo peso al nacer, siendo esencial para justificar políticas estatales de salud más activas en relación con la atención prenatal de calidad.

Palabras clave: Edad materna, Recién nacido, Peso al nacer, Mujeres embarazadas, El parto.

\section{INTRODUÇÃO}

Nas últimas décadas, houve aumento de mulheres que engravidam tardiamente, com incremento de primíparas nessa faixa etária. Dados do Sistema de Informações Sobre Nascidos Vivos (SINASC) referentes ao ano de 2001 mostram que mulheres com 35 anos ou mais tiveram 270.982 filhos nascidos vivos enquanto em 2011 este número foi 317.508, representando aumento de 17,2\% (BRASIL, 2012).

Com o objetivo de sistematizar e agregar conhecimento, o Sistema de Informação de nascidos Vivos (SINASC) foi instituído em 1990 pelo Ministério da Saúde (MS), por meio da Declaração de Nascido Vivo (DNV). As informações contidas nos formulários são variáveis sociodemográficas da genitora, número de consultas no pré-natal, antecedentes obstétricos, duração da gestação, condições socioeconômicas, tipo de parto e características dos Recém-nascidos (RN), como sexo, peso ao nascer e Índice de Apgar. Esses dados são fundamentais para a composição do perfil epidemiológico dos nascidos vivos, dando embasamento para criação e implementação de políticas de saúde voltadas para o núcleo materno-infantil (PEREIRA CCB, et al., 2011).

Dos 15 milhões de bebês que nascem antes do tempo, cerca de um milhão destes morrem dias após o parto. A prematuridade é segunda causa de morte de crianças com menos de cinco anos, ficando atrás apenas da pneumonia. No Mundo, os nascimentos prematuros são responsáveis por $11,1 \%$ em todos os nascidos vivos. O Brasil e os Estados Unidos da América (EUA) figuram entre os dez países com maior número absoluto em nascimentos prematuros. No topo da lista está a Índia com 3.519 .100 casos. Nos EUA, cerca de $12 \%$ dos nascimentos são pré-termos, isso significa dizer que aproximadamente um a cada nove nascidos vivos é prematuro neste país. Em décimo lugar no ranking, o Brasil conta com 279.300 nascimentos prematuros (BRASIL, 2012; OMS 2012).

A OMS define prematuro todo aquele que nasce com menos de 37 semanas. Entretanto, os prematuros não são um grupo homogêneo somente por essa definição, pois o grau de maturidade fisiológica difere com a idade gestacional (IG). Portanto, foi proposta subclassificação mais adequada dividindo os RN pré-termo em: prematuro tardio quando a IG varia entre 32 e 37 semanas incompletas, já os nascidos entre 28 e 32 semanas são ditos muito prematuro, enquanto o prematuro extremo tem menos de 28 semanas (FERREIRA APA, 2011).

O peso ao nascer é um parâmetro utilizado para verificar as condições de saúde do RN. O Baixo Peso ao Nascer (BPN), considerado como peso menor que $2.500 \mathrm{~g}$, está ligado a maior mortalidade e morbidade neonatal e infantil, sendo o fator isolado mais influente na sobrevivência nos primeiros anos de vida. Tendo ou não a IG como referência, a proporção de BPN é um dos indicadores mais importantes para ajuizar a assistência pré-natal (ANDRADE CLT, et al., 2005)

A associação entre a taxa de mortalidade infantil e o BPN é observada em todo o mundo. Acima de $95 \%$ dos RN de baixo peso nascem em países em desenvolvimento. Todavia, os fatores determinantes para 0 baixo peso são diferentes entre os países em desenvolvimento e os desenvolvidos. Nos primeiros, ocorrem pela realidade desfavorável, como baixo nível de instrução dos pais, desemprego, idade materna (CAÇOLA P, BOBBIO TG, 2010).

Segundo dados de 2011 do Departamento de Informática do Sistema Único de Saúde do Brasil (DATASUS), a proporção de nascidos vivos com BPN no Brasil foi de $8,53 \%$. Em comparação às demais regiões do País, a região Norte apresentou menor porcentagem de crianças com BPN, contando com 7,34\%. O Pará teve 4098 RN simultaneamente prematuros e peso inadequado, ao passo que a Região Norte obteve 
10.154 e o Brasil 130.637. A cidade de Belém do Pará teve proporção acima da média da sua região com 9,76\%, sendo $51,9 \%$ destes também prematuros (BRASIL, 2012).

Tendo em vista o papel da prematuridade e BPN na mortalidade infantil no país, em especial nas regiões mais carentes Norte e Nordeste, faz-se necessário identificar quais são os fatores de risco associados ao aumento da incidência de casos através de estudos específicos. Sabe-se que a Região Norte, em especial o Estado do Pará, ocupa o segundo lugar em mortalidade perinatal. Nesse sentido, o objetivo da presente pesquisa foi avaliar os fatores de risco associados à prematuridade e baixo peso ao nascer dos recémnascidos das parturientes nos extremos da vida reprodutiva em uma maternidade privada no estado do Pará.

\section{MÉTODOS}

Trata-se de um estudo transversal, retrospectivo, descritivo e comparativo, com a coleta de dados realizada em uma maternidade privada no estado do Pará, obtendo-se informações dos prontuários das gestantes que pariram, no período de janeiro de 2012 a janeiro de 2013. A coleta de dados foi conduzida durante os meses de janeiro a março de 2014. O tempo total de desenvolvimento deste trabalho foi de novembro de 2013 a abril de 2014.

\section{Participantes}

Participaram da pesquisa todos os registros de mulheres que tiveram partos no período estabelecido, que atendessem aos seguintes critérios de inclusão: faixa etária entre 10 e 19 anos e acima ou igual a 35 anos, dados completamente preenchidos com informações suficientes para o preenchimento da ficha de coleta de dados. $\mathrm{O}$ total de partos ocorrido no período estipulado foi de 4.174. Após a aplicação dos critérios de inclusão e exclusão permaneceram 1.117 prontuários.

As gestantes incluídas no estudo foram subdivididas em dois grupos previamente estabelecidos, a saber: um grupo de gestantes adolescentes (faixa etária entre 10 e 19 anos) e outro de gestantes tardias (mulheres com 35 anos ou mais). Os critérios de exclusão foram: todas as gestantes fora da faixa etária estabelecida, gemelares natimortos e malformações congênitas.

\section{Procedimento de coleta}

Coletou-se os dados dos prontuários e Declaração de Nascido Vivo (DNV) das gestantes e de seus recémnascidos, respectivamente, através de um Protocolo de Pesquisa previamente formulado pelos pesquisadores. Investigou-se 12 variáveis independentes entre si: idade materna; estado civil; grau de escolaridade; paridade (quantificação do número de gestações, partos normais ou cesáreas anteriores e número de abortos); idade gestacional; realização de pré-natal; intercorrências; via de parto; ocupação; Apgar; peso ao nascer e encaminhamento. A pesquisa foi totalmente financiada com recursos próprios.

\section{Análise dos dados}

O software Excel 2010 foi adotado para entrada dos dados, bem como para confecção das tabelas e gráficos, e a análise estatística foi realizada por meio dos softwares Epi Info 3.5.1 e BioEstat 5.0.

Para uma investigação mais detalhada dos fatores de risco possivelmente associados à prematuridade $\mathrm{e}$ ao baixo peso para todo o grupo e separadamente nas duas faixas etárias estudadas, procedeu-se uma estratificação dos dados por duas faixas etárias (10 a 19 anos e de 35 ou mais anos), sendo então empregados na Análise de Variância (ANOVA), o cálculo das Razões de Chances (OR), com significância estatística verificada pelo Teste paramétrico do Qui-Quadrado (X2) e/ou teste exato de Fisher com um nível alfa de 0,05 .

\section{Considerações éticas}

Todos os participantes desta pesquisa foram estudados segundo os preceitos da Declaração de Helsinque e do Código de Nuremberg, respeitadas as Normas de Pesquisas envolvendo Seres Humanos Res. CNS 466/2012 do Conselho Nacional de Saúde (CNS) após aprovação do projeto pelo Comitê de Ética sob o 
número 506.272/2014. A coleta dos dados foi realizada após a obtenção do Termo de Consentimento de Uso de Banco de Dados (TCUD).

\section{RESULTADOS}

Os achados da pesquisa foram organizados da seguinte forma: (1) características sociodemográficas maternas; (2) dados perinatais de acordo com a faixa etária materna; (3) análise bivariada entre os grupos de mães e (4) características perinatais de acordo com a faixa etária materna.

\section{Características sociodemográficas maternas}

Na população de estudo de 1.117 gestantes, observou-se 159 (14,2\%) mães adolescentes e 958 (85,7\%) mães com idade maior ou igual a 35 anos. A média da idade das mães adolescentes (limites de 14 a 19 anos) foi de 17,2 $\pm 1,2$ anos, e a das mães adultas (limites de 35 a 48 anos) foi de $37,4 \pm 2,2$ anos.

Em relação à escolaridade, prevaleceu o nível médio 107 (67,3\%) entre as adolescentes e o nível superior completo $596(62,2 \%)$ entre as mulheres com 35 anos ou mais. Com relação ao estado civil, $110(69,2 \%)$ adolescentes eram solteiras, e $538(56,2 \%)$ mulheres adultas eram casadas. Com relação à paridade, 138 $(86,8 \%)$ adolescentes eram primíparas, contrastando com $656(68,5 \%)$ das mulheres de idade avançada que eram multíparas. Quanto ao número de consultas durante o pré-natal, constatou-se que a maioria das mulheres pesquisadas frequentou da forma idealizada pelo MS, com seis ou mais consultas durante o prénatal, 149 (93,7\%) no grupo das adolescentes e 935 (97,6\%) entre as mães mais velhas.

Quanto à análise de comorbidades que sabidamente poderiam interferir no desfecho como a Doença Hipertensiva Específica na Gravidez (DHEG), Oligoâmnio e Aminiorrexe Prematura, não foi possível computar por não estar descrito em todos os prontuários analisados. No entanto, a proporção foi maior para DHEG (38,23\%), Oligoâmnio (29,41\%) e Aminiorrexe Prematura (17,64\%).

\section{Dados perinatais de acordo com a faixa etária materna}

Ao investigar os dados perinatais de acordo com a faixa materna verificou-se que no grupo de mães com idade entre 14 e 19 anos ( $n=159), 158(99,4 \%)$ eram mães de bebês com peso entre $2500 \mathrm{~g}$ e $4000 \mathrm{~g}$, dos quais $145(91,2 \%)$ eram pré-termos, com registro de Apgar no 5 o minuto acima de 7 em 100\% dos casos. A amostra foi composta por neonatos do sexo masculino em $51,6 \%$ (82) dos casos, encaminhados para 0 Alojamento Conjunto (ALCON) após o nascimento em 91,2\% (145) dos casos, com desfecho de alta em 100\% dos casos. No grupo de mães com idade acima ou igual a 35 anos verificou-se que $829(86,5 \%)$ da amostra era composta por recém-nascidos com peso entre $2500 \mathrm{~g}$ e $4000 \mathrm{~g}$, dos quais $90,8 \%$ eram nascidos a termo, tendo registrado Apgar no $5^{\circ}$ minuto acima de 7 em 99,4\% (952) dos casos. Ademais em $52,9 \%$ (507) dos casos eram bebês do sexo masculino, que foram encaminhados para a ALCON em 91,3\% (875) dos casos, com desfecho de alta em $90,4 \%$ (75) dos casos.

No grupo de mães com idade acima de 35 anos observou-se um total de 958 casos, sendo em $86,5 \%$ mães de neonatos com peso entre 2500 e 4000 gramas, pré termos (90,8\%), com Apgar acima de sete em $99,4 \%$ dos casos. Além disso verificou-se sexo masculino em $52,9 \%$ dos registros, tendo sido encaminhado para a ALCON em $91,3 \%$ dos casos com desfecho de alta em $90,4 \%$ dos casos.

Quanto ao desfecho desfavorável destes neonatos oito crianças evoluíram ao óbito na Unidade de Terapia Neonatal (UTIN), três estavam na faixa de baixo peso $(2490 \mathrm{~g}, 1940 \mathrm{~g}$ e $1610 \mathrm{~g})$, três eram muito baixo peso $(1320 \mathrm{~g}, 1260 \mathrm{~g}$ e $1000 \mathrm{~g})$ e os dois restantes eram de extremo baixo peso $(990 \mathrm{~g}$ e $700 \mathrm{~g})$. A IG variou de 26 a 37 semanas, média de 31,25 semanas, enquanto a idade materna variou entre 35 a 42 anos com médias de 37,5 anos. Não houve óbitos entre os filhos de mães adolescentes.

\section{Análise bivariada entre os grupos de mães e os desfechos prematuridade e BPN}

Quando se comparou as variáveis faixa etária com a chance de nascer prematuro por meio da ANOVA bivariada observou-se que, apesar de não ocorrer significância estatística na presente pesquisa, as mães mais velhas apresentaram maior chance. O mesmo acontecendo quando se analisou o BPN. 


\section{Características perinatais de acordo com a faixa etária materna}

A Tabela 1 mostra as características perinatais de acordo com os dois grupos de mães de faixa etária diferente.

Observou-se que a variável atividade laboral nas mães acima de 34 anos apresentou fator protetor $(O R=0,41)$ e estatisticamente significante $(p=0,0055)$ em relação à prematuridade. No entanto, a variável prénatal menor que seis consultas indicaram maior chance de nascimento prematuro $(\mathrm{OR}=3,67)$ com significância estatística $(p=0,0004)$. Ao analisar o baixo índice de Apgar no 5ำ minuto dos $R N$ de mães com idade avançada, verificou-se uma chance de 10,2 vezes maior de ocorrer parto prematuro $(p=0,0122)$, conforme apresentado (Tabela 1).

Tabela 1 - Análise das características perinatais de acordo com a faixa etária materna e o desfecho prematuridade. Belém-PA, 2016. $(n=1117)$

\begin{tabular}{|c|c|c|c|c|c|c|}
\hline \multirow{3}{*}{ Variáveis } & \multicolumn{3}{|c|}{ Idade Materna } & \multicolumn{3}{|c|}{ Prematuridade } \\
\hline & \multicolumn{3}{|c|}{14 a 19 anos } & \multicolumn{3}{|c|}{35 anos ou mais } \\
\hline & $\mathrm{N}(\%)$ & OR & $\mathrm{p}$-valor & $\mathrm{N}(\%)$ & OR & $\mathrm{p}$-valor \\
\hline \multicolumn{7}{|l|}{ Ocupação } \\
\hline Do lar & $15(13,3 \%)$ & 1 & & $76(18,4 \%)$ & 1 & \\
\hline Atividade Laboral & $41(7,3 \%)$ & 0,51 & 0.4902 & $870(8,5 \%)$ & 0,41 & $0,0055^{*}$ \\
\hline Estudante & $103(8,7 \%)$ & 0,62 & 0.5700 & 12 & - & 0,9718 \\
\hline \multicolumn{7}{|l|}{ Número de consultas } \\
\hline Seis ou mais & $149(8,7 \%)$ & & & $935(8,8 \%)$ & & \\
\hline Menos que seis & $10(10 \%)$ & 1,16 & 0,6133 & $23(26,1 \%)$ & 3,67 & $0,0004^{*}$ \\
\hline \multicolumn{7}{|l|}{ Apgar5' } \\
\hline$<7$ & $159(8,8 \%)$ & & & $6(50 \%)$ & & \\
\hline$\geq 7$ & - & - & 1,0 & $952(8,9 \%)$ & 10,2 & $0,0122^{\prime}$ \\
\hline
\end{tabular}

Teste Qui-quadrado/Exato de Fisher.

Fonte: Chermont AG, et al., 2019.

No grupo das mães com idade igual ou maior a 35 anos, a atividade laboral também funcionou como fator protetor para o BPN, com significância estatística $(p=0,0370)$. Ainda em relação à mesma idade materna, 0 número de consultas inferior a seis, na presente pesquisa, representa uma chance de quase 3,5 vezes de nascer baixo peso. No que tange a variável Apgar no 5 minuto menor que sete, predominou na mesma faixa etária com chance de nascer baixo peso 20 vezes maior $(p=0,0010)$. No entanto, a chance de nascer com baixo peso prevaleceu em ambas as faixas etárias nos bebês do sexo feminino, com uma tendência para as mães adolescentes. Quanto ao desfecho, ainda em relação ao BPN, observou-se significância estatística para o óbito dos filhos de mães com 35 anos ou mais $(p=0,0076)$, conforme observado (Tabela 2). 
Tabela 2 - Análise das características perinatais de acordo com a faixa etária materna e o desfecho baixo peso ao nascer ( $n=1117)$. Belém-PA, 2016.

\begin{tabular}{|c|c|c|c|c|c|c|}
\hline \multirow{3}{*}{ Variáveis } & \multicolumn{3}{|c|}{ Idade Materna } & \multicolumn{3}{|c|}{ Baixo Peso ao Nascer } \\
\hline & \multicolumn{3}{|c|}{14 a 19 anos } & \multicolumn{3}{|c|}{35 anos ou mais } \\
\hline & $\mathrm{N}(\%)$ & OR & $\mathrm{p}$-valor & $\mathrm{N}(\%)$ & OR & p-valor \\
\hline \multicolumn{7}{|l|}{ Ocupação } \\
\hline Do lar & $15(6,7 \%)$ & 1 & & $76(17,1 \%)$ & 1 & \\
\hline Atividade Laboral & $41(4,9 \%)$ & 0,71 & 0,7931 & $870(9,1 \%)$ & 0,45 & $0,0370^{*}$ \\
\hline Estudante & $103(7,8 \%)$ & 1,17 & 0,8809 & 12 & - & 0,9666 \\
\hline \multicolumn{7}{|l|}{$\mathrm{N}$ de consultas } \\
\hline Seis ou mais & $149(6,7 \%)$ & \multirow{2}{*}{1,54} & \multirow{2}{*}{0,5223} & $935(9,2 \%)$ & \multirow{2}{*}{3,48} & \multirow{2}{*}{$0,0066^{*}$} \\
\hline Menos que seis & $10(10 \%)$ & & & $23(26,1 \%)$ & & \\
\hline \multicolumn{7}{|l|}{ Apgar5' } \\
\hline$<7$ & $159(6,9 \%)$ & \multirow{2}{*}{ - } & \multirow{2}{*}{1,0} & $6(66,7 \%)$ & \multirow{2}{*}{19,6} & \multirow{2}{*}{$0,0010^{*}$} \\
\hline$\geq 7$ & - & & & $952(9,2 \%)$ & & \\
\hline \multicolumn{7}{|l|}{ UTI-Prognóstico } \\
\hline Alta & $15(40 \%)$ & \multirow{2}{*}{ - } & \multirow{2}{*}{1,0} & $75(50,7 \%)$ & - & \multirow{2}{*}{$0,0076^{*}$} \\
\hline Óbito & - & & & $8(100 \%)$ & & \\
\hline
\end{tabular}

Teste Qui-quadrado/Exato de Fisher

Fonte: Chermont AG, et al., 2019.

\section{DISCUSSÃO}

A presente pesquisa mostrou que o número de gestantes adolescentes $(3,8 \%)$ foi menor do que aquelas com idade superior ou igual a 35 anos $(22,95 \%)$ e que esses resultados foram diferentes ao registrado no DATASUS do ano de 2011 (19,25\% e 10,83\%, respectivamente). Essa diferença pode ser explicada pelo nível socioeconômico e cultural do público atendido no serviço estudado.

Sobre a escolaridade, as adolescentes, em sua maioria, tinham ensino médio, já no outro grupo predominou o ensino superior completo. Apenas uma publicação revelou que o grau de escolaridade das adolescentes foi maior que das mulheres com 35 ou mais. O grau de escolaridade influencia diretamente na qualidade de cuidados que a gestante deve tomar a partir da concepção assim pode haver consequências desfavoráveis como a baixa qualidade do pré-natal, a alimentação e possíveis vícios inadequados durante a gestação (SAAS A, et al., 2011; RAMOS HAC, CUMAN RKN, 2009).

Quanto à situação conjugal, estudos realizados com adolescentes revelam pequeno índice de casamentos formais, predominando uniões consensuais. Outras publicações mostram prevalência de solteiras na fase da adolescência semelhante aos dados da presente amostra (adolescentes solteiras 69,2\% (SAAS A, et al., 2011; HARVILLE EW, 2012).

Quanto ao número de consultas durante o pré-natal, observou-se que quase $100 \%$ das pacientes realizou assistência adequada (93,7\% entre as adolescentes e 97,6\% entre as gestantes mais velhas) levando-se em consideração o tempo de gestação, como preconizado pelo Ministério da Saúde. Este achado não condiz com os resultados de outras publicações, que mostram percentuais inferiores, principalmente entre as adolescentes. Entretanto, outros estudos demonstraram índices médios de $97 \%$ das mulheres do estudo realizaram pré-natal completo, bem semelhante à presente pesquisa (MARTINS MG, 2011; GRAVENA AAF, 2013; SANTOS GHN, 2009; GALVÃO MCB, DAVIN RNB, 2011). 
Com relação ao tipo de parto, a frequência do parto operatório foi de quase $100 \%$ nas duas faixas etárias estudadas, muito superiores ao encontrado na literatura. Diferente do estudo de coorte de nascimentos realizado em uma cidade do Rio Grande do Sul que demonstrou que as cesáreas representaram $45 \%$ dos partos, bem abaixo do resultado encontrado nesta pesquisa. A OMS recomenda uma proporção de $15 \%$ de partos cesáreos do total de partos realizados. O parto operatório atualmente representa $43 \%$ dos partos realizados no Brasil. No setor privado, esse percentual é ainda maior, chegando a $80 \%$. Estima-se que o Brasil realize cerca 560.000 cesarianas consideradas desnecessárias. Entre os fatores que sugerem a opção por um parto operatório, têm sido declarados: a organização da atenção obstétrica, pautada pela conveniência de uma intervenção programada; fatores institucionais ligados à forma de pagamento e anulação do pagamento de anestesia peridural para partos vaginais pelo Sistema Único de Saúde (SUS). Tais situações evidenciam uma importante distorção no modelo de atenção ao parto, submetendo mães e crianças a riscos desnecessários (BARROS AJD, 2004; MARTINS MG, et al., 2011; FARIA DGS, ZANETTA DMT, 2008; CUNHA MA, et al., 2002; LIMA EFA, et al., 2012).

O peso ao nascer ainda é utilizado como importante indicador de sobrevivência de RN de risco, por alguns autores, sendo o principal problema para a saúde pública, e um indicador global de saúde que traduz a eficiência do sistema de saúde local (MAGALHÃES MC, CARVALHO MS, 2003).

$\mathrm{Na}$ análise da variável BPN, verificou-se a ocorrência de 6,36\% de RN entre as mães adolescentes e 9,6\% entre as mães tardias. Os valores encontrados no presente estudo estão abaixo do encontrado em outras literaturas, onde o BPN, com frequência, é maior que $10 \%$ nas duas faixas etárias, por vezes ultrapassando 20\%. O resultado encontrado provavelmente é devido a um melhor índice socioeconômico, maior acessibilidade ao pré-natal e melhor índice educacional. Contudo, a incidência de BPN foi superior no grupo de gestantes tardias. Os principais dados na literatura estudada direcionam o BPN a favor da gestante adolescente. A discordância deve-se ao fato das mães com 35 anos ou mais apresentarem comorbidades (DHEG, Aminiorrexe Prematura e Oligoâmnio) proporcionalmente maior do que as mães adolescentes, favorecendo tais resultados encontrados na presente pesquisa (SAAS A, et al., 2011; SANTOS GHN, et al., 2009; ANDRADE PC, et al.,2004; CASTELL EC, et al., 2013).

A presente pesquisa demostrou a importância da idade materna como fator de risco para BPN e para mortalidade infantil, particularmente entre as adolescentes com menos de 20 anos e entre as mães com 35 anos e mais. O estudo conduzido em Camarões demonstrou que as mulheres com idade avançada têm maior chance de seus bebês apresentarem BPN, Índice de Apgar menor que sete, assim como o número maior de internações na UTIN e mortalidade perinatal (LIMA EFA, et al., 2012; NGOWA JDK, et al., 2013).

Todos os filhos de mães adolescentes tiveram índice de Apgar maior que sete, enquanto o índice inferior a sete entre as mães tardias foi de 0,6\%. Estes dados, apesar de inferiores aos encontrados na literatura, apontam pior índice de Apgar no quinto minuto nas gestantes tardias. Estudo retrospectivo ocorrido na capital do estado do Ceará, com 11.127 nascidos vivos, evidenciou um percentual de 1,4\% inferior a sete no quinto minuto, valor muito próximo ao encontrado pelo presente estudo (SILVA CF, et al., 2006).

\section{CONSIDERAÇÕES FINAIS}

A contribuição da presente pesquisa foi mostrar que o pré-natal incompleto, com menos de seis consultas foi uma variável constante nas duas faixas etárias estudadas para prematuridade e baixo peso ao nascer. As mães com idade avançada tiveram um maior número de partos prematuros e filhos com baixo peso ao nascimento, e os fatores relacionados a esses desfechos foram: número de consultas menor que seis, índice de Apgar menor que sete no quinto minuto e atividade laboral. O desfecho para óbito na UTIN foi maior entre os recém-nascidos com baixo peso ao nascer de mães com idade avançada. A limitação do presente trabalho deveu-se à obtenção retrospectiva dos dados e o fato de não ter se obtido informações a respeito de comorbidades durante a gestação (Hipertensão Arterial, DHEG, Diabetes mellitus, entre outras), provavelmente mais frequentes no grupo de gestantes com 35 anos ou mais e que possuem repercussão diretas nas variáveis estudadas. Portanto recomenda-se mais estudos de forma prospectiva e com outras instituições hospitalares a fim de confirmar ou confrontar os achados da presente pesquisa. 


\section{REFERÊNCIAS}

1. ANDRADE CLT, SZWARCWALD CL, CASTILLO EA. Baixo peso ao nascer no Brasil de acordo com as informações sobre nascidos vivos do Ministério da Saúde, 2005. Cad. Saúde Pública. 2008; 24(11): 2564-2572. DOI: http://dx.doi.org/10.1590/S0102-311X2008001100011.

2. ANDRADE PC, et al. Resultados perinatais em grávidas com mais de 35 anos: estudo controlado. Rev Bras Ginecol Obstet. 2004; 26(9):697-701. DOI: http://dr.doi.org/10.1590/S0100-72032004000900004.

3. BARROS AJD, et al. Coorte de nascimentos de Pelotas, 2004: metodologia e descrição. Rev Saúde Pública. 2006; 40(3):402-13. DOI: http://dx.doi.org/10.1590/S0034-89102006000300007.

4. BRASIL. Ministério da Saúde. Departamento de Informática do SUS. Informações de saúde (TABNET) [Internet]. Estatísticas Vitais; 2012.

5. BRASIL. Ministério da Saúde. Datasus Informações de saúde. Taxa de mortalidade infantil [Internet]. 2011

6. CASTELL EC, et al. Maternal age as risk factor of prematurity in Spain; Mediterranean área. Nutr Hosp. 2013; 28(5): 1536-1540. DOI: 10.3305/nh.2013.28.5.6500.

7. CAÇOLA P, BOBBIO TG. Baixo peso ao nascer e alterações no desenvolvimento motor: a realidade atual. Rev. paul. pediatr. 2010; 28(1): 70-6.

8. CUNHA MA, et al. Gestação na Adolescência: Relação com o Baixo Peso ao Nascer. Rev Bras Ginecol Obstet. 2002; 24(8): 513-519.

9. FARIA DGS, ZANETTA DMT. Perfil de mães adolescentes de São José do Rio Preto/Brasill e cuidados na assistência pré-natal. Arq Cienc Saúde. 2008; 15(1);17-23.

10. FERREIRA APA, ALBUQUERQUE RC, RABELO ARM, et al. Comportamento visual e desenvolvimento motor de recém-nascidos prematuros no primeiro mês de vida. Rev. bras. crescimento desenvolv. hum. 2011; 21(2): $335-343$.

11. GALVÃO MCB, DAVIM RNB. Perfil de puérperas internadas em um hospital. Rev enferm UFPE online. $2011 ; 5(7): 1591-595$.

12. Gravena AAF, Paula MG, Marcon SS, Carvalho MDB, Peloso, SM. Idade materna e fatores associados a resultados perinatais. Acta Paul Enferm. 2013; 26(2): 130-5. DOI: http://dx.doi.org/10.1590/S0103-21002013000200005.

13. GUANABENS MFG, GOMES AM, MATA ME, et al. Gravidez na adolescência: um desafio a promoção da saúde integral do adolescente. Rev Bras Educ Med. 2012; 36(2): 20-24. DOI: http://dx.doi.org/10.1590/S010055022012000300004.

14. HARVILLE EW, MADKOUR AS, XIE Y. Predictors of birth weight and gestacional age among adolescents. Am J epidemiol. 2012; 176(7): 150-63. DOI: 10.1093/aje/kws231.

15. LIMA EFA, et al. Perfil de nascimentos de um município: um estudo de coorte. Rev Bras Pesq Saude. 2012; 14(1): 12-18. DOI: https://doi.org/10.21722/rbps.v0i0.3404.

16. MAGALHÃES MC, CARVALHO MS. Atenção hospitalar perinatal e mortalidade neonatal no município de Juiz de Fora, Minas Gerais. Rev Bras Saúde Mater Infantil. 2003; 3(3): 329-37. http://dx.doi.org/10.1590/S151938292003000300012.

17. MARTINS MG, et al. Associação de gravidez na adolescência e prematuridade. Rev Bras Ginecol Obstet. 2011; 23(11): 354-60. DOI: http://dx.doi.org/10.1590/S0100-72032011001100006.

18. NGOWA JDK, et al. Pregnancy outcome at advanced maternal age in a group of African women in two teaching Hospitals in Yaounde, Cameroon. Pan African Medical Journal. 2013; 14: 134. DOI: 10.11604/pamj.2013.14.134.2315.

19. ORGANIZAÇÃO MUNDIAL DA SAÚDE. 15 milhões de bebês nascidos muito cedo [Internet]. 2012.

20. PEREIRA CCB, et al. Avaliação da implantação do Sistema de Informações sobre Nascidos Vivos (SINASC) em Pernambuco. Rev Bras Saude Mater Infant. 2013; 13(1): 39-49. DOI: http://dx/doi.org/10.1590/S151938292013000100005.

21. PORTAL BRASIL. Brasil está entre os dez países com o maior número de partos prematuros [Internet] 2012.

22. REIS SLS, et al. Parto normal X Parto cesáreo: análise epidemiológica em duas maternidades no sul do Brasil. Rev AMRIGS. 2009; 53 (1): 7-10.

23. RAMOS HAC, CUMAN RKN. Fatores de risco para prematuridade: pesquisa documental. Esc Anna Nery Rev Enferm. 2009; 13(2): 297-304. DOI: http://dx.doi.org/10.1590/S1414-81452009000200009.

24. SAAS A, et al. Resultados perinatais nos extremos da vida reprodutiva e fatores associados ao baixo peso ao nascer. Rev Gaúcha Enferm. 2011; 32(2): 352-8. DOI: http://dx.doi.org/10.1590/S1983-14472011000200020.

25. SANTOS GHN, et al. Impactos da idade materna sobre os resultados perinatais e via de parto. Rev Bras Ginecol Obstet. 2009; 31(7): 326-34.

26. SILVA CF, et al. Fatores de risco para mortalidade infantil em município do Nordeste do Brasil: linkage entre bancos de dados de nascidos vivos e óbitos infantis: 2000 a 2002. Rev Bras Epidemiol. 2006; 9(1): 69-80). DOI: http://dx.doi.org/10.1590/S1415-790X2006000100009.

27. SILVA JLCP, SURITA FGC. Idade materna: resultados perinatais e via de parto. Rev. Bras. Ginicol. Obstet. 2009; 31(7): 321-5. DOI: http://dx.doi.org/10.1590/S0100-72032009000700001. 